\title{
Psychological Review of the Book, 'Wintergirls'
}

\author{
Aprajita Dixit* and Aditi Seth \\ Department of Mental Health and Life Skills Promotion, Moolchand Medcity, Delhi, India
}

Email of corresponding author: aprajitadixit18@gmail.com

\begin{abstract}
Lia Overbrook (M.C.) and Cassie Parrish have been best friends since the third grade, but as they grow up, both develop destructive obsessions with body image. Lia is anorexic, while Cassie is a victim of bulimia, constantly trying to bring each other down and when things get too tough in the months before their senior year in high school, Cassie breaks off the friendship, leaving Lia devastated. Six months later, after not having communicated with her ex-friend in ages, Lia receives thirty-three phone calls from Cassie in one evening, but she refuses to answer. Next day, Lia receives the news of her best friend's death due to excessive puking alone in an isolated motel room, Lia realizes that Cassie had been calling for help, and she is wrecked by guilt. Lia keeps her parents, along with her father's new wife, at a distance, concealing her inner troubles and maintaining an appearance of normalcy. Lia's family is worried that it might trigger her and she might fall back to old habits, which she does.
\end{abstract}

Keywords: Eating Disorder; Anorexia; Self Harm; Mental Illness; Losing Weight; Treatment.

\section{DISORDER PICTURED}

\section{Anorexia Nervosa}

It is an eating disorder manifested when a person refuses to eat an adequate amount of food or is unable to maintain the minimal weight for a person's body mass index. Individuals with anorexia often have a distorted body image. They have an intense fear of gaining weight or becoming fat. The diagnostic criteria for Anorexia Nervosa according to DSM 5:

A. Restriction of energy intake relative to requirements leading to a significantly low body weight in the context of age, sex, developmental trajectory, and physical health. Significantly low weight is defined as a weight that is less than minimally normal or, for children and adolescents, less than that minimally expected.

B. Intense fear of gaining weight or becoming fat, or persistent behaviour that interferes with weight gain, even though at a significantly low weight.

C. Disturbance in the way in which one's body weight or shape is experienced, undue influence of body weight or shape on self-evaluation, or persistent lack of recognition of the seriousness of the current low body weight.

\section{CHARACTERISTIC FEATURES}

Lia didn't start with a low body weight but as the story progresses, she continues to lose weight at a rapid pace and it soon falls below the number that was deemed as a safe level for her. She feels that she is strong because she was able to resist the temptation of eating and was able to achieve her body weight goals. She felt "stronger" when her body weight was lower than the weight that was healthy for her according to her age

She started to losing weight and she reached one of her weight goals. However, she herself acknowledges that however much weight she might lose, she will have another goal and will not be happy

Lia has unrealistic weight goals and body standards After cassie's death, she starts to avoid eating, using tricks to make it seem like she ate when she actually didn't, or eat very small amounts that contain exactly the amount of calories she knows she needs to function in daily life. Lia also exercised her body to the point of exhaustion, much more than what her body needed.

While she was losing weight, Lia also showed signs of depressed mood. For example she said "I wish I had cancer" at one point. She is also more irritable with her family and also had insomnia. There are instances in the book describing the vivid somatic symptoms that she experienced during these periods of insomnia, like spiders crawling all over her. She once binged and because she could not control herself in that point of time, she later took several laxatives. As a result, she had extreme stomach pains for an entire night. 


\section{EFFECTS OF THE}

\section{CONDITION ON LIA AND} FAMILY MEMBERS

- Lia sees Cassie's ghost at night, telling her to continue with her habits and to join her soon.

- Lia's psychologist tells that Lia's mind created Cassie's ghost because she was depressed. She offers a rational explanation for events that had occurred. Lia although continues to believe that she is haunted.

- She cuts herself, distances herself from family, and lives with extreme selfdeprecating thoughts and nightmares about Cassie.

- Towards the end her body weight was extremely low and she had no energy.

- She was having symptoms like dizziness, black spots in vision and generally her body was physically not healthy

- She worries that she will kill someone while she is driving and not know it because she is in a starvation-induced trance.

- She dreams about crows eating her body and thinks about stabbing herself with a knitting needle and bleeding out in the snow.

- Because of her refusal to eat, life becomes increasingly tense at her father and stepmother's home. Lia fights with her father about whether she should be admitted for treatment.

- When the ghost of Cassie does not leave her alone, Lia foes as far as to cut her chest with a sharp knife, but Emma (stepsister that she loves) witnesses this and so she does not die.

- In the end of the story, in the hotel, starving and perilously close to a sleeping pill overdose, Lia fades in and out of consciousness.

- She toys with thoughts of suicide.
- Her father, David, cries on her mattress when he visits her every day at the hospital.

- It is implied that Cassie's parents' high expectations contributed to her eating disorder.

\section{MANAGEMENT}

Lia was admitted to an institution called new seasons. She had been getting therapy since the first time she was admitted, she had a major breakthrough however only in the end. This time, she wanted to recover, and she works to slowly rebuild her fractured relationship with her family. She still sees Cassie's ghost, but it doesn't try to talk to her anymore, and she knows that Emma wants her to come back home.

Treatment: The main goal of treatment of anorexia nervosa is to restore your body to a normal weight and establish normal eating habits. A dietitian helps learn how to eat properly. Individual, family, and group therapies are often an integral part of treatment.

Hospitalization: The hospital admits the individual for a few days to treat the effects of your anorexia nervosa. They may be put on a feeding tube and intravenous fluids if their weight is too low or if they're dehydrated.

Therapy: Cognitive behavioural therapy (CBT) helps change unhealthy thoughts and behaviours. Its goal is to help learn to cope with strong emotions and build healthy self-esteem. Family therapy gets family members involved in keeping the individual on track with healthy eating and lifestyle. Family therapy also helps resolve conflicts within the family.

\section{REFERENCES}

[1] Wintergirls Book review by Debra Bogart, Common Sense Media. https://www.commonsensemedia.org/book-reviews/wintergirls

[2] Laurie Halse Anderson. https://madwomanintheforest.com/book/wintergirls/

(C) 2020 Aprajita Dixit and Aditi Seth; Licensee ATSK Publishers.

This is an open access article licensed under the terms of the Creative Commons Attribution Non-Commercial License (http://creativecommons.org/licenses/by-nc/3.0/) which permits unrestricted, noncommercial use, distribution and reproduction in any medium, provided the work is properly cited. 